\title{
1868 : Le moment Cro-Magnon
}

\section{8: The Cro-Magnon Moment}

\author{
A. Hurel \\ Reçu le 02 juillet 2018; accepté le 25 septembre 2018 \\ (C) Société d'Anthropologie de Paris et Lavoisier SAS 2018
}

Résumé Le gisement paléolithique de Cro-Magnon et ses fossiles humains érigés au rang de type selon les usages raciologiques, ont rapidement acquis une renommée considérable. Ils fournissaient aux préhistoriens un ensemble cohérent de matériaux et une abondance de restes humains d'une haute antiquité incontestable. Toutefois, ces événements, canonisés au cours des décennies suivantes par l'historiographie internaliste, méritent à cent cinquante ans de distance un nouvel examen. Cet article se propose donc de restituer à ces découvertes leur contexte historique et épistémologique, mais aussi de les analyser dans leur dimension d'altérité. En effet, les discussions autour de l'âge de la sépulture de Cro-Magnon interrogent la capacité, voire la volonté, de la communauté scientifique à accepter dans son entièreté l'univers des Préhistoriques qui se dévoile alors à eux.

Mots clés raciologie $\cdot$ indice céphalique $\cdot$ sépultures paléolithiques $\cdot$ culture

\begin{abstract}
The Paleolithic deposit at Cro-Magnon and its human fossils, which were raised to the status of a "racial type" in accordance with usage at the time of their discovery, soon gained considerable renown. They provided prehistorians with a coherent set of materials and an abundance of human remains of unquestionable antiquity. However, although canonized in the following decades by internalist historiographers, these circumstances deserve to be reexamined at a distance of one hundred and fifty years. This article proposes to restore the discoveries to their historical and epistemological context, but also to offer an analysis in terms of their "otherness": indeed, the discussions around the age of this Cro-Magnon burial raise the question of the capacity, and even the willingness, of the scientific commu-
\end{abstract}

\footnotetext{
A. Hurel

Département « Homme Environnement » du Muséum national d'Histoire naturelle (UMR 7194, UMR 8560)
}

nity to accept, in its entirety, the prehistoric world that these discoveries revealed to them.

Keywords raciology $\cdot$ cephalic index $\cdot$ paleolithic burials culture

\section{Introduction}

Le 5 août 1868 meurt Jacques Boucher de Perthes (17881868), celui qui incarnait l'acceptation du principe de haute antiquité de l'Homme. Ses travaux, menés dans les carrières d'Abbeville depuis deux décennies, avaient obtenu en 1859 la reconnaissance officielle attendue par lui après avoir été confortés par d'autres recherches conduites en France et en Angleterre. Une question majeure recevait alors réponse : il y a plusieurs milliers d'années, l'Homme fut bien contemporain de grands mammifères aujourd'hui éteints ou disparus. Ses outils, des pierres taillées, associés à des ossements fossiles dans des couches profondes de terrasses alluviales en portent témoignage.

Cette accumulation de preuves indirectes attendait confirmation par la mise au jour d'ossements humains, ceux de l'artisan de ces industries, dans les mêmes contextes archéologiques et géologiques. La mandibule de Moulin Quignon (1863) ne pouvait qu'imparfaitement remplir cet office en raison des doutes exprimés à son égard. Ce sont donc d'autres trouvailles moins suspectes d'être attachées à une supercherie, tout comme un regard neuf porté sur des pièces signalées antérieurement qui apportèrent une contribution décisive.

Les galeries de l'histoire du travail de l'Exposition universelle de Paris de 1867 offrirent à la préhistoire une consécration officielle et publique. Quelques mois plus tard, à la fin du mois de mars 1868, des travaux de génie civil menés dans le bourg des Eyzies (Dordogne), à proximité de la gare de chemin de fer, amènent la découverte fortuite de plusieurs squelettes humains rapidement rapportés à l'âge du renne. Aussitôt dépêché sur place par le ministre de l'Instruction publique, Louis Lartet (1840-1899) opère une fouille. Le 
gisement se signale à la fois par la richesse de ses niveaux archéologiques (les squelettes de quatre adultes et un enfant, plusieurs centaines de coquilles de littorines percées, des bois de renne travaillés, des pierres taillées, des charbons, etc.), mais tout autant par sa portée anthropologique. En effet, Lartet interprète nombre de ces menus objets comme autant d'éléments de parure et, surtout, s'affirme convaincu que ce lieu fut une halte de chasse puis une habitation et finalement une sépulture.

Rapidement, le site de Cro-Magnon et ses fossiles humains, érigés au rang de type selon les usages raciologiques, acquièrent une renommée considérable. Ils fournissent aux préhistoriens un ensemble cohérent de matériaux et une abondance de restes humains d'une haute antiquité incontestable, la découverte ayant été faite dans un abri celé par un talus d'éboulements de plus de 4 mètres d'épaisseur. Dans le grand public, ce nom de Cro-Magnon fait florès et deviendra même à lui seul quasiment synonyme de la préhistoire et des Préhistoriques.

Toutefois, ces événements, canonisés au cours des décennies suivantes par l'historiographie internaliste, méritent à cent cinquante ans de distance un nouvel examen. Cet article se propose donc de restituer à ces découvertes leur contexte historique et épistémologique, mais aussi de les analyser dans leur dimension d'altérité. En effet, les discussions autour de l'âge de la sépulture de Cro-Magnon interrogent la capacité, voire la volonté, de la communauté scientifique à accepter dans son entièreté l'univers des Préhistoriques qui se dévoile à eux.

\section{La haute antiquité de l'Homme est un principe acquis}

\section{L'acception du principe de haute antiquité de l'Homme}

Dans la première partie du $\mathrm{XIX}^{\mathrm{e}}$ siècle, les recherches autour de la question de l'homme des origines connaissent un fort développement. Elles sont menées concomitamment au sein de deux champs disciplinaires distincts (sciences naturelles, sciences humaines) et selon des méthodologies propres. Qu'il s'agisse de mettre en lumière des associations stratigraphiques d'ossements d'une faune disparue ou éteinte avec des artéfacts lithiques, ou d'interpréter des outils à l'aune du comparatisme, les publications et les recherches sur le terrain, du Sud-Ouest de la France à la Scandinavie en passant par l'Angleterre et la Belgique, sont nombreuses. Ces travaux construisent un cadre général permettant de penser les temps antéhistoriques à travers l'esquisse de chronologies archéologiques (« système des trois âges ») ou paléontologiques.

Par convention historiographique, l'année 1859 est considérée comme celle de la réunion et consécration de ces deux axes de recherche grâce au consensus international sur l'acceptation de la haute antiquité de l'Homme. Cette année-là, naturalistes et archéologues anglais et français s'accordent à valider dans les carrières des terrasses alluviales de la Somme des observations effectuées antérieurement d'associations de pierres taillées avec des faunes fossiles, dans des niveaux stratigraphiques profonds et non remaniés. Ainsi, Amiens, Abbeville et, outre-Manche, Brixham Cave apporteraient la même démonstration, les mêmes preuves indirectes, de l'existence de l'Homme dans des temps antéhistoriques. Si l'Académie des sciences peine à reconnaître l'événement dans toutes ses dimensions, la Société géologique de France [1] et la jeune Société d'anthropologie de Paris [2] saluent sans retenue cette conclusion. Mais c'est à l'occasion de la séance du 15 septembre 1859 du congrès d'Aberdeen de l'Association britannique pour l'avancement des sciences que la consécration arrive, lorsque le géologue Charles Lyell (1797-1875) annonce alors sa conversion à la Préhistoire et à l'homme préhistorique.

Le grand bénéficiaire de ce moment de " cristallisation », pour reprendre le terme opportunément choisi par Annette Laming-Emperaire (1917-1977) [3], est sans conteste Jacques Boucher de Perthes (1788-1868) qui, depuis plus d'une décennie, tentait de faire reconnaître son homme antédiluvien et ses " silex ouvrés ». Bien qu'au cœur d'un vaste réseau scientifique, national et international, il n'avait jusque-là recueilli au mieux que de la sympathie à l'égard de ses travaux, marqués par « un curieux mélange de divagations et de vérités » $[3: 120]$. Cette fois, son archéo-g éologie, même grevée d'un encombrant fatras métaphysique, semble répondre aux attentes et à la nécessaire synthèse entre archéologie, géologie et paléontologie stratigraphique.

En 1860, le paléontologue Édouard Lartet (1801-1871) peut démontrer que les indices permettant de prouver l'ancienneté géologique de l'espèce humaine sont maintenant parfaitement établis. Ils reposent, d'une part, sur la présence de « silex taillés, offrant des formes définies et évidemment intentionnelles » associés à des « restes d'espèces animales éteintes » dans des couches non remaniées permettant de conclure à leur " association consécutive », leur « communauté d'origine » et leur « synchronisme de préexistence » et, d'autre part, sur la possible mise en évidence de « traces non équivoques d'une action humaine quelconque sur les os mêmes des animaux enfouis avec les silex travaillés de main d'homme » $[4: 118]$.

Sa conviction est si fermement établie que, lorsqu'il annonce à l'Académie des sciences (21 août 1865) la découverte dans le gisement de la Madeleine d'un mammouth gravé sur ivoire, Lartet ne peut que constater que « ce nouveau fait n'ajoutera rien aux convictions déjà acquises que la coexistence de l'Homme avec l'Éléphant fossile (Elephas primigenius) et les autres grands Herbivores ou Carnassiers que les géologues considèrent comme ayant vécu dans les 
premières phases de la période quaternaire. Cette vérité d'évidence rétrospective se déduit aujourd'hui d'un si grand nombre d'observations concordantes et de faits matériels d'une signification tellement manifeste, que les esprits les moins préparés à l'admettre ne tardent pas à l'accepter dans toute sa réalité, dès qu'ils veulent bien prendre la peine de voir, et, après cela, de juger en conscience. » $[5: 310-311]$

Le professeur d'anthropologie du Muséum d'histoire naturelle Armand de Quatrefages (1810-1892) conclut lui aussi que « la présence d'ossements humains dans une couche du globe n'est nullement nécessaire pour faire admettre que notre espèce vivait à l'époque de la formation de cette couche. Il est évident que les traces de son industrie suffisent en pareil cas. » $[6: 177]$

\section{Des ossements humains comme preuve directe}

Le nouveau cadre épistémologique validé autour de 1859 et des travaux de Boucher de Perthes amène à examiner différemment les découvertes, voire à reconsidérer des travaux précédents (par un nouveau regard porté sur des publications et des collections). La séquence en vallée de la Somme clôt les questionnements sur la haute antiquité de l'Homme mais ouvre la voie à la confirmation et à la généralisation. De chaque coin de France des faits similaires viennent affermir les données acquises. Toutefois, la preuve directe, celle des " caractères ethniques », c'est-à-dire des ossements humains mis au jour dans un contexte stratigraphique permettant d'en déduire l'ancienneté géologique, reste attendue tout en étant superfétatoire puisque la question de la haute antiquité de l'Homme est maintenant résolue. Cette découverte ressortit alors à d'autres registres heuristiques, comme l'ethnogénie et l'anthropométrie.

Dès 1837, au moment de présenter sa découverte du Pliopithecus, Édouard Lartet exprimait sa conviction que " l'existence paléontologique de l'homme » n'avait rien «d'invraisemblable à [ses] yeux » [7:159]. Comme le précise Goulven Laurent (1925-2008), « Ce concept n'a rien à voir, en soi, avec celui de son ascendance simienne, comme on a pu le croire trop facilement. Ce que Lartet soutient et démontre, c'est que l'Homme est aussi ancien que le Singe, et que tous deux sont aussi anciens que les autres fossiles que l'on trouve dans les couches géologiques. » $[8: 27]$ En effet, Lartet insère ses vues dans la perspective d'une création des espèces vivantes, et donc de l'homme, unique et complète : «Que sait-on si des observations ultérieures ne viendront pas tôt ou tard nous apprendre que cette nature ancienne, encore si peu connue, n'était ni moins complète, ni moins avancée dans l'échelle organique que celle de l'époque actuelle ? " [9:96]

Lartet va transformer en système chronologique l'ensemble des données acquises peu à peu sur la présence des grands mammifères quaternaires dans les gisements. Il fonde sa démonstration sur leur distribution géographique et leur position stratigraphique selon un certain nombre de sites. Ses observations le conduisent à proposer une classification ordonnée par la succession dans le temps des espèces. Il définit quatre époques caractérisées chacune par une espèce typique : « Nous aurions ainsi, pour la période de l'humanité primitive, l'âge du grand Ours des cavernes, l'âge de l'Éléphant et du Rhinocéros, l'âge du Renne, et l'âge de l'Aurochs, à peu près comme les archéologues ont récemment adopté les divisions de l'âge de la pierre, de l'âge du bronze et de l'âge du fer. » $[10: 231]$

Il reprend ainsi un principe classique de la paléontologie stratigraphique qui veut que les diverses formations géologiques soient individualisées par les fossiles caractéristiques qu'elles contiennent et leur ordre de superposition.

En 1861, son étude du site d'Aurignac lui permet d'insérer dans cette chronologie l'ensemble du matériel, dont des restes humains, mis au jour dans l'abri ou dans le remblai extérieur : «En employant la méthode archéologique, on trouve dans l'absence de toute espèce de métal, et dans l'emploi usuel d'outils et d'armes de silex et d'os, des indications suffisantes pour faire remonter les circonstances de cette station d'Aurignac à cette période ancienne des temps antéhistoriques, que les antiquaires désignent aujourd'hui sous le nom d'âge de la pierre. Par la méthode paléontologique, la race humaine d'Aurignac se classerait dans le plus haut degré d'ancienneté où l'on ait jusqu'à présent constaté la présence de l'Homme ou des débris de son industrie. En effet, cette race a été évidemment contemporaine de l'aurochs, du renne, du cerf gigantesque, du rhinocéros, de l'hyène, etc., mais encore du grand Ours des cavernes (Ursus spelaeus), qui paraît être [...] l'espèce la plus anciennement disparue de ce groupe de grands mammifères, que l'on invoque toujours comme caractéristiques de la dernière période géologique. » $[10: 201]$

La découverte d'ossements humains à Aurignac conduit Lartet à rappeler des recherches antérieures qui auraient pu permettre d'attester plus tôt la contemporanéité de l'Homme avec des espèces disparues, qu'il s'agisse des travaux de Paul Tournal (1805-1872) menés à Bize entre 1827 et 1834, de Jules de Christol (1802-1861) dans le Gard publiés en 1829 [11], mais aussi ceux de Philippe-Charles Schmerling (1790-1836) du côté de Liège à Engis dans les années 1833-1834 ${ }^{1}$. Au-delà de ces études anciennes et incomplètement reconnues en leur temps ${ }^{2}$, plusieurs découvertes de restes humains viennent, dans la foulée des événements de 1859, préciser la connaissance de l'homme fossile.

En 1858, le marquis Paul de Vibraye (1809-1878) fait exécuter des fouilles dans la grotte des Fées d'Arcy-

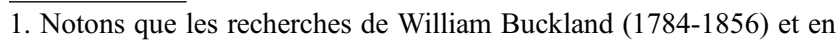
particulier la Red Lady of Paviland ne sont pas évoqués.

2. Sur Tournal et la question préhistorique voir Hurel [12].
} 
sur-Cure. Il s'emploie à mettre en évidence la stratigraphie. L'année suivante, il distingue une portion de mandibule humaine au milieu d'ossements d'Ursus spelaeus, de Hyena spelaea, de Rhinoceros tichorhinus extraits de la couche inférieure du dépôt. Le 16 avril 1860, lorsqu'il présente ses résultats à ses collègues de la Société de géologie, Vibraye doit composer avec le scepticisme d'une partie d'entre eux à l'égard de ses conclusions géologiques et paléontologiques. Certains font ainsi remarquer que la grotte a dû souvent être atteinte par les crues d'un cours d'eau proche, produisant des mélanges dans le dépôt archéologique, et que Vibraye n'a pas lui-même assisté au dégagement de la mâchoire dans le sédiment [13].

Cette question de l'administration de la preuve de l'authenticité des découvertes va être au cœur de l'affaire de la mandibule humaine mise au jour à Abbeville en 1863 dans les carrières de Moulin Quignon. Contrairement au consensus de 1859, savants britanniques et français ne vont pas s'accorder sur les conclusions à tirer. Les uns, comme Joseph Prestwich (1812-1896), John Evans (1823-1908) ou Hugh Falconer (1808-1865) vont douter puis dénoncer une fraude dont Boucher de Perthes aurait été la dupe, trompé par les carriers. D'autres, tels Quatrefages ou Lartet, vont soutenir la validité intégrale de la découverte. L'examen précis du dossier montre que les discussions ne portèrent pas sur la portée phylogénétique des ossements humains mais sur la valeur des pierres taillées dégagées dans les mêmes niveaux stratigraphiques. Celles-ci apparaissaient suspectes tant dans leur abondance que dans leur aspect parfois peu conforme aux caractéristiques des industries lithiques alors reconnues [14]. La préhistoire qui est en train de créer sa légitimité dans le concert des sciences institutionnalisées ne peut alors s'encombrer de doutes.

L'épisode controversé de Moulin Quignon se trouve finalement marginalisé par la profusion des recherches qui viennent remplir le blanc de la carte européenne des gisements paléolithiques.

\section{La problématique anthropologique}

\section{L'occasion d'une rencontre totale avec les Préhistoriques}

La lecture des périodiques scientifiques et des bulletins des sociétés savantes donne l'impression qu'après 1859 l'Europe continentale est devenue un vaste chantier de prospections. Ce moment de l'histoire de la préhistoire, qui porte reconnaissance d'un nouveau champ de recherche, a ouvert la voie spécifique d'une archéologie du tout un chacun qui, peu ou prou, va perdurer en France jusqu'à la Seconde Guerre mondiale [15]. Elle se différencie des domaines classiques de l'archéologie entre autres par ses acteurs individuels ou institu- tionnels, ses prérequis scientifiques quasiment nuls et ses méthodes puisant tout autant du côté des sciences naturelles que de l'archéologie. Ces caractéristiques favorisent la multiplication des initiatives sur le terrain, qu'il s'agisse de ramassages d'objets en surface ou de fouilles, et la constitution de nombreuses collections dans lesquelles l'art mobilier de l'« âge du renne » fait office de chef-d'œuvre.

Les noms d'Édouard Lartet et Henry Christy (1810-1865) dominent cette production, à la fois par le caractère systématique de leurs prospections en vallée de la Vézère, par la richesse de leurs récoltes et la qualité de leurs publications ${ }^{3}$. Toutefois, d'autres travaux vont avoir pour effet de confronter les préhistoriens à l'ensemble des pratiques, aujourd'hui qualifiées de symboliques, des Préhistoriques, dont au premier chef la mise à jour de sépultures paléolithiques.

La découverte des restes humains dans l'abri de CroMagnon n'est pas juste une question d'anatomie des Préhistoriques. En effet, plusieurs facteurs conduisent tout de suite les observateurs à porter un regard différent sur ces restes : le nombre des squelettes, la diversité d'âge et de sexe des individus, leur association avec de nombreux menus objets rapidement assimilés à des éléments de parures. Tout ceci concourt à donner un statut particulier au gisement, interprété comme une sépulture, et donc légitimement aux Préhistoriques eux-mêmes, qui apparaissent à la fois porteurs d'une complexité sociale et, en définitive, d'une certaine proximité avec l'univers de leurs inventeurs. La mort et le soin apporté aux défunts par les vivants font partie intégrante de la vie, des codes et usages sociaux de l'homme du XIX ${ }^{\mathrm{e}}$ siècle.

Dès sa présentation à la réunion annuelle des sociétés savantes à la Sorbonne, le 17 avril 1868, Louis Lartet évoque le principe d'une « sépulture des anciens Troglodytes du Périgord » [16]. L'emploi de ce terme de sépulture interroge. Si le Nouveau dictionnaire de la langue française (1856) de Pierre Larousse la définit sobrement comme le « lieu où l'on enterre », en revanche son utilisation par les anthropologues et préhistoriens recouvre tout autant l'idée du lieu où gisaient les restes humains fossilisés qu'une notion implicite de spiritualité, voire de religiosité. Il est vrai que la démonstration d'Édouard Lartet concernant le site d'Aurignac associait aux observations archéologiques et paléontologiques les notions de « rites funéraires », « cérémonies religieuses ou funéraires », « consécration funéraire » etc. [10]

Sa conviction que les pratiques funéraires s'ancrent dans la profondeur de l'histoire de l'Homme va se conforter au fil de ses recherches. Se fondant sur la « sépulture primordiale

3. Il s'agit en particulier de leurs Reliquiae aquitanicae dont les premiers des dix-sept fascicules commencent à paraître en décembre 1865. Les textes rédigés en anglais sont d'une grande rigueur scientifique et bénéficient de l'excellence et du nombre des planches du volume d'atlas. L'ensemble a un impact fort sur la communauté internationale naissante des préhistoriens. 
d'Aurignac » où l'on peut voir « professer une sorte de culte des morts », sa conviction est que les sépultures et les lieux d'habitation étaient distincts et que des dépôts (armes, " objets d'affection ", ornements, " os de mammifères exceptionnellement non fragmentés »...) accompagnaient le corps du défunt [17]. Cette certitude se nourrit également des résultats d'autres recherches en Belgique et en France.

À partir de 1864, le géologue Édouard-François Dupont (1841-1911) mène des fouilles en Belgique dans plus d'une vingtaine de grottes des bords de la Lesse. Quatre d'entre elles livrent des restes humains en nombre (Trou des Nutons, Trou du Frontal, Trou Rosette à Furfooz, Trou de Chaleux), mais c'est la mandibule découverte dans le Trou de la Naulette qui, par sa conformation (région mentonnière verticale, branches épaisses etc. [18]), va retenir toute l'attention - le professeur de médecine Paul Broca (1824-1880), non darwiniste, estime même que celle-ci « est le premier fait qui fournisse un argument anatomique aux darwinistes. C'est le premier anneau de la chaîne qui doit, suivant eux, s'étendre de l'homme au singe » $[19: 595]$ - et favoriser des comparaisons avec la mâchoire d'Arcy-sur-Cure. Mais, plus globalement, Dupont cherche à se livrer à une analyse totale des Préhistoriques, dont il a trouvé en abondance artefacts et ossements. Son ambition est de « reconstituer les caractères de ces hommes, leur race, leur industrie, leurs mœurs et même quelques-unes de leurs coutumes » [20:4]. Il bénéficie pour cela de l'aide de Carl Vogt (1817-1895), Édouard Van Beneden (1846-1910), George Busk (1807-1886), Armand de Quatrefages, Édouard Lartet et Franz PrunerBey (1808-1882), qui est alors l'anthropologue référent pour de multiples expertises. Pour Dupont, l'homme de l'âge du renne des bords de la Lesse était un chasseur appartenant au " grand rameau Touranien », connaissant l'usage du feu, disposant d'une industrie lithique et osseuse simple et sans ornementation, ayant le goût de la parure (coquilles fossiles et ossements perforés, collecte de fluorine). Ces populations auraient aussi été fétichistes, superstitieuses et auraient marqué « leur respect pour les morts par les soins qu'elles donnent à leur sépulture, et les objets, sans doute ceux qui étaient chers au défunt, placés dans la cavité funèbre, montrent clairement leur croyance à une autre vie. » [20:73]

Ces découvertes, auxquelles s'ajoutent celles de Bruniquel en 1864 [20] puis à partir de 1866 celles de Solutré [22] [23], confortent les conclusions antérieures de Lartet sur le caractère sépulcral d'Aurignac et le décorum funéraire qu'il a cru y déceler. Ce qu'Émile Cartailhac (1845-1921) appellera la " poésie d'Aurignac » [24] prend rapidement une place incontournable dans la littérature scientifique. Aurignac devient le site de référence concernant les pratiques funéraires au Paléolithique. Charles Lyell (17971875), par exemple, dans The Geological evidence of the antiquity of man, se fondant sur les observations de Lartet estime que si celles-ci sont justes « we have at last succeeded in tracking back the sacred rites of burial, and, more interesting still, a belief in a future state, to times long anterior to those of history and tradition. Rude and superstitious as may have been the savage of that remote era, he still deserved, by cherishing hopes of a hereafter, the epithet of "noble", which Dryden gave to what he seems to have pictured to himself as the primitive condition of our race. » $[25: 193]^{4}$

Cette démonstration conforte l'opinion de chercheurs comme Quatrefages, l'un des acteurs majeurs de l'anthropologie française. Cette mise en lumière de pratiques funéraires concorde avec sa défense du principe d'existence d'un " règne humain » dans la nature. Selon lui, « l'Homme est le seul être chez lequel se rencontrent les trois faits fondamentaux suivants : $1^{\circ}$ la notion du bien et du mal moral ; $2^{\circ}$ la croyance à une autre vie ; $3^{\circ}$ la croyance à des êtres qui lui sont supérieurs. Ces deux derniers, parfois difficiles à distinguer l'un de l'autre, peuvent être rapportés à une même faculté, la religiosité. Le premier se rattache à la moralité. Ces deux facultés sont pour [lui] les attributs du Règne humain. » 6 : 76] Les découvertes faites dans l'abri CroMagnon appuient sa certitude que s'il est « bien difficile de juger des caractères religieux et surtout moraux d'un peuple éteint [...] il est à peu près certain que ces premiers habitants de l'Europe croyaient à une vie future. ». En définitive, le Préhistorique est fondamentalement proche " de bien des peuples aujourd'hui existants sur divers points du globe. » [27:26]

Toutefois, les débats en cours autour de la théorie mongoloïde et de la brachycéphalie supposée des Préhistoriques vont occulter en 1868 la question de la sépulture, et subséquemment orienter les représentations sur l'univers symbolique des hommes de l'âge de la pierre. Pourtant, cette même année, Gabriel de Mortillet (1821-1898) se rendant à Solutré avait admis les preuves que lui présentaient Henri TestotFerry (1826-1869) et Adrien Arcelin (1838-1904) : «Les diverses sépultures du Clos du Charnier, à Solutré, me paraissent bien être contemporaines des débris de silex, de renne et de chevaux, qui ne forment qu'un tout indivisible. Disciple fervent, j'ai même dépassé mes maitres. Et au lieu de voir dans les foyers de Solutré des débris de cuisine et d'habitations, je crois que le Clos du Charnier est simplement et purement un cimetière. C'est une station des morts et non une station des vivants, ce qui est beaucoup plus intéressant, car jusqu'à présent nous n'en connaissions pas de cet âge et de cette importance. » $[28: 324]$

\footnotetext{
4. «Alors nous avons enfin réussi à retrouver dans le passé la trace des cérémonies funèbres, et, ce qui est bien plus intéressant encore, nous avons constaté la croyance en une vie future, à des temps bien antérieurs à ceux de l'histoire et de la tradition. Tout grossiers et superstitieux qu'aient été les sauvages de cet âge reculé, ils nourrissaient l'espoir d'une autre vie et méritaient l'épithète de « nobles » que donne Dryden à l'homme primitif qu'il semble s'être représenté comme le type originel de notre race. » $[26: 200]$
} 
Quatre ans plus tard, lors de la présentation à la Société d'histoire naturelle de Toulouse de la découverte de l'« homme écrasé » de Laugerie-Basse, Émile Cartailhac exprime ses doutes sur les « sépultures dites de l'âge du renne » (Bruniquel, Solutré, Cro-Magnon). Son travail de révision de ces sites le conduit à contester l'ancienneté effective des squelettes et de leur association chronologique avec les niveaux dans lesquels ils reposaient. Lartet aurait avec le site d'Aurignac, devenu la référence, biaisé les recherches futures en les orientant vers un modèle impossible : «L'influence du mémoire sur Aurignac a été, en effet, considérable sur la direction des études préhistoriques, et les explorateurs étaient surtout à la recherche de stations identiques. De fait, leurs vœux furent souvent comblés, quelques fois déçus. » [29: 207]

À Cro-Magnon, s'il s'agit bien d'une sépulture ancienne, en raison de l'épaisseur du talus qui la recouvrait, « Il est très possible qu'elle ne soit pas beaucoup plus récente que le dépôt sous-jacent, mais il est difficile d'être certain qu'elle est quaternaire. Il ne faut pas oublier que le gisement des squelettes humains était fouillé à l'arrivée de M. Louis Lartet, et par conséquent des observations importantes peuvent nous manquer. » [29:214] En 1886, Cartailhac opèrera un revirement sur cette question des sépultures paléolithiques en s'appuyant sur la multiplication des cas, en particulier dans les grottes de Grimaldi ${ }^{5}$, et les enseignements tirés du comparatisme ethnographique ${ }^{6}$. Mais, à cette date, il sera bien seul. En effet, sa prise de position radicale de 1872 a entrainé derrière lui Mortillet et, avec celui-ci, tout le parti du matérialisme scientifique.

Le 28 avril 1876, lors d'une discussion à la Société d'anthropologie de Paris, Mortillet intervient en arguant du travail de révision des grottes opéré par Cartailhac pour corriger l'âge de la sépulture de Cro-Magnon : « II ne faut pas trop se baser sur l'ancienneté de Cro-Magnon. Tout d'abord, entraîné par l'opinion générale, j'ai été, comme beaucoup d'autres, porté à croire que les hommes de Cro-Magnon dataient des temps les plus reculés. De nouvelles études, faites avec plus de réflexion et de soins, m'ont conduit à un avis contraire. Le gisement de Cro-Magnon, malheureusement, n'a pas pu être étudié scientifiquement. Il a été découvert par des ouvriers qui travaillaient au chemin de fer, et exploité par M. Laganne, un simple chercheur. Lorsque M. Louis

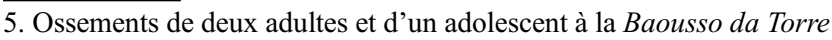
en 1873 , squelettes de deux enfants à la Grotte des enfants en 1874 et 1875 et celui d'un adulte à la Barma Grande en 1884.

6. Dans son manuel, La France préhistorique (1896), Cartailhac consacrera un chapitre au " culte des morts dans les cavernes et les stations quaternaires " soutenu par une étude d'ethnographie comparée sur les « rites funéraires des sauvages actuels ». Le site de Cro-Magnon est présenté dans ce livre à côté de ceux de Solutré, Menton, Sordes, Bruniquel, Gourdan, Laugerie-Basse, les Eyzies, Raymonden, Spy.
Lartet y est allé, toute la sépulture était explorée depuis plusieurs jours. Ses recherches n'ont pu porter que sur l'ensemble du gisement et sur les couches inférieures. De toutes les observations faites, de ma propre inspection des lieux, il résulte que la grotte-abri de Cro-Magnon a été habitée pendant un long espace de temps, tout au commencement de l'époque magdalénienne. Cette époque y a laissé un puissant dépôt riche en ossements d'animaux et en objets d'industrie, silex et os travaillés, bien caractéristiques. C'est sur ce dépôt, ou tout au moins à son sommet, qu'on a trouvé les sépultures, puis venaient des éboulis plus ou moins récents. Comme on le voit, la sépulture peut tout au plus être rapportée à la fin de l'occupation magdalénienne du gisement ; mais elle peut et paraît être plus récente encore. C'était évidemment une sépulture ; or nous ne connaissons point de sépultures certaines de l'époque magdalénienne, tandis que celles de l'époque de la pierre polie abondent dans les grottes. » [30 : 184-185]

Lorsqu'il s'agira pour Mortillet de rédiger l'entrée « CroMagnon (paléoethnologie) »du Dictionnaire des sciences anthropologiques, il précisera que « Tout d'abord on crut être en présence d'une sépulture quaternaire ; mais un examen plus calme et plus complet des lieux a montré que c'était une sépulture robenhausienne, faite dans un milieu plus ancien. Les corps déposés dans la petite grotte, on avait fermé l'entrée avec du magdalénien remanié. » [31:357] Or, ce point de vue est conforme avec le courant dominant de l'anthropologie dans le dernier quart du $\mathrm{XIX}^{\mathrm{e}}$ siècle qui dénie toute religiosité à l'homme quaternaire, celle-ci importée d'Orient étant envisagée comme l'apanage de la période néolithique.

\section{Les cadres conceptuels de l'anthropologie}

Au milieu de $\mathrm{XIX}^{\mathrm{e}}$ siècle, le positionnement de la préhistoire s'opère dans un contexte d'émergence de l'anthropologie en tant que questionnement scientifique de l'espèce - selon Michel Foucault (1926-1984) le XIX ${ }^{\mathrm{e}}$ siècle serait celui de l'apparition réelle de l'Homme, des sciences de l'Homme en raison d'une conscience épistémologique nouvelle de l'Homme $^{7}$ - et en tant qu'espace disciplinaire identifié et militant. De fait, Armand de Quatrefages, loue dans son rapport de 1867 au ministre de l'Instruction publique le mouvement collectif, intellectuel et institutionnel, de développement que l'anthropologie connait en France [33]. Le Dr Ernest-Théodore Hamy (1842-1908) note pour sa part en

\footnotetext{
7. «Avant la fin du XVIII ${ }^{\mathrm{e}}$ siècle, l'homme n'existait pas. Non plus que la puissance de la vie, la fécondité du travail ou l'épaisseur historique du langage. C'est une toute récente créature que la démiurgie du savoir a fabriquée de ses mains, il y a moins de deux cents ans : mais il a si vite vieilli, qu'on a imaginé facilement qu'il avait attendu dans l'ombre pendant des millénaires le moment d'illumination où il serait enfin connu. » [32:319]
} 
1868 que la partie de l'anthropologie qui a enregistré récemment les plus importants progrès est la «paléoethnologie ou anthropologie préhistorique.» $[34: 280]$

Pour Quatrefages, cette anthropologie est globale, collective et individuelle, naturelle et culturelle, présente et passée. Elle doit embrasser « l'examen comparatif des organes et celui des fonctions, l'étude des variations que présente le type fondamental, celle des instincts et des mœurs. En d'autres termes, le naturaliste moderne étudie les êtres vivants à tous les points de vue possibles, soit comme individus, soit comme espèce. » [6:1] Broca envisage " l'anthropologie générale » comme « la biologie du genre humain. » [35: 300], avec pour objet « l'étude du groupe humain considéré dans son ensemble, dans ses détails et dans ses rapports avec le reste de la nature. » $[35: 276]$

Le grand débat qui agite l'anthropologie à cette époque est d'ordre taxonomique, puisqu'il concerne la réalité des principes d'unité ou de multiplicité de l'espèce humaine, monogénisme ou polygénisme. La diversité, qu'établissent alors de façon normative un peu plus chaque jour les anthropologistes, semble poser directement la question de l'existence d'une ou plusieurs espèces humaines. Cette variété somatique exprimerait selon les uns des « caractères de race»" au sein de l'espèce, pour les autres des " caractères d'espèce ». La définition des termes est nécessaire et en l'occurrence décisive. Quatrefages souligne en 1867 l'hétérogénéité des principes retenus pour la détermination de la notion d'espèce. Depuis Tournefort jusqu'à Carl Vogt en passant par Buffon, Lamarck ou Cuvier, les points de vue sont tranchés mais tous ont de commun de reconnaître « comme caractères essentiels de l'espèce la filiation et la ressemblance. » [6 : 105] Dans cette perspective, un écart taxonomique constitué et relevé à la mesure d'un groupe, puis établi dans sa descendance constituerait, selon Quatrefages, une race. Ce terme recouvrirait donc selon lui la variabilité constatée au sein de l'espèce humaine. D'autres, au contraire, comme Broca verront dans les races, établies sur les caractères physiques de l'homme et dont l'anthropométrie rend compte, la définition de types cohérents et fixes ${ }^{8}$. Quelles que soient les positions doctrinales des uns et des autres, la race devient le critérium. Elle est à la fois une méthode, une fin et une explication au service d'une anthropologie raciologique.

Un autre trait contextuel de cette anthropologie est à prendre en considération dans la perspective des débats sur la préhistoire de l'Homme. En 1867, dans son Rapport sur les progrès de l'anthropologie, Quatrefages ne cache pas les problèmes de tendances qui traversent la communauté. Les allusions à cet égard sont nombreuses et pas toutes cryptiques. Les sciences de l'Homme, par l'ontologie qu'elles questionnent ou dont elles sont porteuses, interagissent avec

8. Une évaluation des ambiguïtés de Broca et des ajustements opérés par lui après 1870 est proposée par Blanckaert [36] d'autres univers intellectuels car « à cette époque marquée par les premiers ralliements à Darwin et le renouveau de l'esprit voltairien, l'anthropologie empiète sur le terrain de la politique passionnelle et de la religion. » $[33: 272]$ Même si les scientifiques français ne semblent faire qu'un usage distant ou approximatif de la théorie darwinienne, celle-ci se trouve mobilisée par le courant matérialiste comme une espèce de bélier à même d'ébranler l'ordre scientifique, politique et religieux [37] [38].

\section{Cro-Magnon : Un individu autochtone ou allochtone ?}

Polygénisme, monogénisme, races, espèces, transformisme ${ }^{9}$, fixité, philosophie, politique constituent les termes et fixent le cadre d'action de l'anthropologie française au milieu du XIX ${ }^{\mathrm{e}}$ siècle. C'est donc dans cet espace sémantique et programmatique que les découvertes faites dans l'abri Cro-Magnon prennent place. Elles deviennent également un enjeu du principe de classification binaire du genre humain entre races dolichocéphales et brachycéphales alors majoritairement en vogue chez les anthropologues.

Cette partition est héritée des travaux d'Anders Adolf Retzius (1796-1860) qui fit de la mesure du rapport de la largeur du crâne avec sa longueur conjuguée à d'autres caractères un attribut à la fois discriminant entre les individus et directeur au plan de l'histoire des races. Ces travaux, présentés en 1842 à l'Assemblée des naturalistes scandinaves de Stockholm, paraissent en France en 1846 [40] où ils connaissent un grand succès jusqu'à devenir « une norme descriptive, en donnant une assise apparemment objective au comparatisme anthropologique » [41:166]. L'humanité présente et passée se voit ainsi divisée, sur un fondement anthropométrique reposant sur un nombre fini de mesures, entre dolichocéphales et brachycéphales, chacune de ces branches étant elle-même subdivisée entre populations orthognathes et prognathes, ce que Retzius synthétise en un tableau qu'il affinera au cours du temps. Ainsi, par exemple, les Gaulois, Celtes, Bretons, écossais, Germains, Scandinaves se trouvent rattachés au même groupe dolichocéphale orthognathe, tandis que les Tartares, Kalmouks, Mongols, Incas et Papou rejoignent celui des brachycéphales prognathes. En Europe, une race autochtone brachycéphale aurait été supplantée par des populations indo-européennes dolichocéphales ayant l'usage des métaux.

Les approximations et la complexité du système mis au point par Retzius entrainèrent des erreurs de compréhension mais ne furent que marginalement des obstacles à sa diffusion [41]. Réduit bien souvent à une opposition

9. L'usage du terme de transformisme renvoie justement à la non introduction du darwinisme en France au $\mathrm{XIX}^{\mathrm{e}}$ siècle liée à des oppositions scientifiques ou idéologiques et à une certaine forme d'incompréhension de la teneur réelle de la théorie de Darwin [39]. 
taxonomique entre dolichocéphales et brachycéphales, ce dispositif va devenir une clef commune d'interprétation ethnogénique.

Son compatriote suédois l'archéologue Sven Nilsson (1787-1883) appliquant les théories de Retzius leur offrit une dimension ethnographique [42]. Il expose que les premiers habitants de la Scandinavie n'étaient pas des dolichocéphales mais des brachycéphales chasseurs et pêcheurs. Cette " race autochtone " aurait été remplacée ultérieurement par des dolichocéphales agriculteurs et d'origine gothique. La troisième période d'occupation, d'origine celtique, serait arrivée du nord et de l'est et aurait introduit le bronze. D'une quatrième phase, aux temps historiques, serait issue la majorité des populations actuelles de Scandinavie. Ce principe de la brachycéphalie originelle des peuples européens s'imposa peu à peu pour les peuples antiques et protohistoriques puis se trouva étendu par certains anthropologues, comme Franz Pruner-Bey, aux Paléolithiques, même en l'absence d'éléments crâniens : «C'est en pleine connaissance de cause que j'insiste sur l'existence d'une race préceltique, brachycéphale et allophylétique, en me fondant sur des mâchoires inférieures et d'autres parties du squelette, sans avoir à ma disposition, dans tous les cas, des crânes entiers. Il existe ici toute une série de faits à l'appui de ma thèse. Et d'abord, grâce à la découverte de $\mathrm{M}$. Dupont, nous pouvons suivre au complet ce type crânien jusqu'à l'époque du renne. En nous reportant ensuite à une époque plus ancienne, celle des grands pachydermes, nous avons pour point de repère la célèbre mâchoire découverte par $M$. de Vibraye dans la caverne d'Arcy, à la plus profonde assise, dans un terrain non remanié, où elle était associée aux ossements de ces animaux. Je mets un moule de cette mâchoire sous vos yeux. Vous y constaterez avec moi que cette pièce petite et épaisse, à dents également petites, à courbe parabolique, à menton droit et arrondi, appartient assurément à la catégorie des précédentes et qu'elle nous autorise à lui attribuer le même type crânien. En est-il autrement des fragments osseux humains et notamment des mâchoires, pièces les plus authentiques qui existent, qui furent découvertes par le père de la paléontologie humaine, M. E. Lartet, à Aurignac, accompagnées du même cortège paléontologique ? Et enfin, si nous descendons avec notre illustre collègue, $\mathrm{M}$. Boucher de Perthes, au fond du diluvium, est-ce que la mâchoire de Moulin-Quignon, et tous les fragments osseux humains recueillis dernièrement par le vénérable fondateur de l'archéologie humaine, nous dénoncent autre chose qu'une race de petite taille et à crâne brachycéphale ? » [43:469-470]

Pour Pruner-Bey, cette brachycéphalie relie cette population antéhistorique, «par ses caractères ostéologiques et trichologiques, autant que par ses coutumes, son mode de vivre et par son idéologie qui nous est dépeinte dans les restes de son langage » [44:455], aux peuples actuels du nord de l'Europe et de l'Asie, tout comme aux Amérindiens.
À l'occasion de la séance du 30 août 1867 du Congrès international d'anthropologie et d'archéologie préhistoriques de Paris, les congressistes souhaitent établir un état des connaissances sur les caractères anatomiques des Préhistoriques et s'interrogent sur la réalité d'une succession en Europe occidentale de plusieurs races. Cette discussion conduit Broca à dresser un bilan des théories mais aussi à exposer son propre point de vue et donc son opposition aux thèses de son confrère Pruner-Bey. S'il reconnait à Retzius le mérite d'avoir fait de la question ethnogénique un sujet d'anthropologie, en revanche il lui reproche d'avoir exagéré l'importance des notions de dolichocéphalie et brachycéphalie, tout comme de les avoir trop généralisées. Pour Broca, tous les progrès enregistrés dans la connaissance de l'homme fossile viennent contredire ce schéma par trop simpliste et s'opposent aux positions de PrunerBey. Broca ne conteste pas l'usage des critères de dolichocéphalie et de brachycéphalie mais dans une perspective polygéniste et autochtoniste plus que comme marqueurs chronologiques. Cette double dimension explique la diversité entre les peuples actuels tout comme celle à l'« âge de la pierre ». Les crânes d'Engis, de Lahr, d'Eguisheim, de Bruniquel et de Néandertal - dans lequel Pruner-Bey persiste à reconnaître un Celte -, dont l'ancienneté géologique est globalement acquise, sont marqués par leur dolichocéphalie. Il est vain de continuer à soutenir, en contradiction avec les faits établis par l'archéologie et au profit d'une cause contestée, que « la population primitive de l'Europe était partout brachycéphale, et que tous les crânes dolichocéphales préhistoriques provenaient de la race aryenne ou indoeuropéenne » $[45: 381]$.

L'examen des restes humains de Cro-Magnon va donner une actualité à cet antagonisme doctrinal et se solder au cours d'âpres discussions qui se tiennent à la Société d'anthropologie de Paris pour l'essentiel lors des séances des 21 mai, 4 juin, 18 juin, 16 juillet 1868. Le dialogue entre Broca et Pruner-Bey est impossible, celui-ci se trouve isolé sur le plan doctrinal, suspecté d'affinités monogénistes, et dans son désir de voir dans le vieillard de Cro-Magnon un représentant des peuples mongoloïdes [45]. Au contraire, pour Broca, les individus de Cro-Magnon, aux crânes dolichocéphales et à la grande taille, apportent la démonstration tangible et irréfragable de l'existence d'un peuple aborigène à la civilisation élevée : « Les troglodytes des Eyzies furent donc barbares, comme tous les hommes de leur temps, et ne nous étonnons pas que ces conditions de leur existence aient laissé de fortes empreintes sur leurs squelettes. Mais ces barbares étaient intelligents et perfectibles, et, tout en continuant leurs luttes contre la nature et contre l'homme lui-m ême, ils surent se créer des loisirs suffisants pour accroître leurs connaissances, pour développer leur industrie et, plus encore, pour s'élever jusqu'à la culture des arts. Des aptitudes aussi précieuses, rares à toutes les époques, mais 
vraiment extraordinaires eu égard au temps où elles se sont manifestées, ne pouvaient éclore qu'à la faveur d'une belle organisation cérébrale, dont nous avons retrouvé l'expression morphologique sur les crânes de la race des Eyzies. » $[47: 390]$

\section{Conclusion}

La découverte du gisement de Cro-Magnon est un moment essentiel de la geste de la préhistoire du XIX ${ }^{\mathrm{e}}$ siècle. Toutefois, elle n'a alors pas contribué à une réelle rupture épistémologique, à tout le moins à proportion de ce qu'a été son appropriation par le grand public et la littérature de vulgarisation. Sa contribution, telle que l'analyse de la production scientifique de l'époque permet de le percevoir, est de conforter et d'étendre géographiquement des certitudes acquises antérieurement sur l'antiquité de l'Homme mais aussi de faire un sort à la théorie mongoloïde et à l'idée d'un peuplement autochtone brachycéphale. La souche originelle nationale française aurait donc été dolichocéphale, de belle stature et de mœurs supérieures ; l'homme de CroMagnon en constitue le type racial.

Le succès de Cro-Magnon repose sur sa richesse archéologique, et au premier chef le nombre inattendu de squelettes humains dégagés, sur son insertion dans les débats anthropologiques en cours, mais aussi sur une communication rapide et efficace de Louis Lartet. En effet, moins de trois semaines après la découverte, le matériel et les premiers résultats de la fouille sont présentés aux délégués des sociétés savantes réunis à la Sorbonne en présence du ministre de l'Instruction publique. Les débats qui se tiennent ensuite à la Société d'anthropologie de Paris connaissent un grand écho et le congrès international de Norwich-Londres d'août 1868 vient parachever la démonstration.

Cependant, la découverte de Cro-Magnon ne se transforme pas en occasion d'ouvrir de discussions sur la question des sépultures, c'est-à-dire sur l'interprétation des pratiques symboliques des Préhistoriques, réduites alors au seul art mobilier de l'âge du renne que Lartet et Christy ont efficacement contribué à faire connaître. Sans doute convient-il de voir ici comme un effet de lot : Cro-Magnon se doit d'être accepté dans sa globalité et non dans l'examen sourcilleux de ses détails. Il règle un débat anthropologique au profit du courant polygéniste et, de manière implicite, flatte l'orgueil national par la belle image qu'il construit des premiers Français. Au-delà, il ouvre la voie à un discours de simplicité qui conforte une perspective raciologique au sein de laquelle le courant du matérialisme scientifique va être un élément moteur jusqu'au début du $\mathrm{XX}^{\mathrm{e}}$ siècle.

La non prise en compte de tout l'univers mental des Préhistoriques peut paraître d'autant plus paradoxale que, sur le plan intellectuel, tous les éléments étaient en réalité réunis pour une rencontre réussie. La session de 1868 à LondresNorwich du Congrès international d'anthropologie et d'archéologie préhistoriques offrait une telle opportunité. C'est au cours de cette réunion que le gisement de Cro-Magnon fut présenté à la communauté internationale par Louis Lartet et discuté par Paul Broca, c'est à ce moment-là également que des avancées conceptuelles sensibles sont esquissées. Il s'agit en particulier de la question de l'évolution qui, pour la première fois, est explicitement liée aux temps préhistoriques à travers la perspective d'un évolutionnisme culturel. Lors de la deuxième séance du congrès, l'anthropologue britannique Edward Burnett Tylor (1832-1917) développa l'idée d'une similitude de vie entre les Préhistoriques et les sauvages actuels, tant sur le plan des techniques que des croyances religieuses. Il établit un lien direct entre celles-ci et les rites funéraires, considérant que la présence manifeste d'offrandes dans les sépultures préhistoriques impliquerait une forme de croyance aux esprits [48]. Pourtant, cette démonstration fondée sur le comparatisme ethnographique ne trouva aucun écho immédiat dans les réflexions des anthropologues et préhistoriens français, sans doute trop occupés à régler leurs problèmes raciologiques et à célébrer une découverte exceptionnelle.

Liens d'intérêts : l'auteur déclare ne pas avoir de liens d'intérêts.

\section{Références}

1. Gaudry A. (1859-1860) Discussion sur les haches trouvées dans le diluvium, près d'Amiens. Bulletin de la Société géologique de France 17: 17-9.

2. Pouchet G. (1859) Sur les débris de l'industrie humaine, attestant l'existence d'une race d'hommes contemporaine des animaux perdus (question de l'homme fossile). Bulletin de la Société d'Anthropologie de Paris 1(1): 42-7

3. Laming-Emperaire A. (1964) Origines de l'archéologie préhistorique en France. Éditions A. et J. Picard, Paris, 239 p.

4. Lartet É. (1860) Sur l'ancienneté géologique de l'espèce humaine dans l'Europe occidentale. Annales des sciences naturelles - Zoologie 14: 117-22.

5. Lartet É. (1865) Lettre relative à une lame d'ivoire fossile trouvée dans un gisement ossifère du Périgord, et portant des incisions qui paraissent constituer la reproduction d'un Éléphant à longue crinière. Comptes rendus hebdomadaires des séances de l'Académie des sciences (61): 309-11

6. Quatrefages A. de (1867) Rapport sur les progrès de l'anthropologie, Recueil de rapports sur les progrès des lettres et des sciences en France. Imprimerie impériale, Paris, 570 p.

7. Lartet É. (1837) Sur les débris fossiles trouvés à Sansan et sur les animaux antédiluviens en général. Comptes rendus hebdomadaires des séances de l'Académie des sciences 5: 158-9.

8. Laurent G. (1993) Édouard Lartet (1801-1871) et la paléontologie humaine, Bulletin de la Société préhistorique française 90 (1): 22-30 
9. Desnoyers J. (1836-1837) Lecture d'une lettre d'Édouard Lartet. Séance du 30 janvier 1837. Bulletin de la Société géologique de France 8: 92-6

10. Lartet É. (1861) Nouvelles recherches sur la coexistence de l'homme et des grands mammifères fossiles réputés caractéristiques de la dernière période géologique. Annales des sciences naturelles - Zoologie $4^{\mathrm{e}}$ série 15: 177-253

11. Christol J. de (1929) Notice sur les ossements humains fossiles des cavernes du département du Gard ; par M. de Christol, secrétaire de la Société d'histoire naturelle de Montpellier ». Annales des mines 5: 517-30

12. Hurel A. (2011) “La géologie commence là où l'archéologie s'arrête" Paul Tournal, les grottes de Bize et la question de la haute antiquité de l'homme. Hurel A., Coye N. (ed) Dans l'épaisseur du temps. Archéologues et géologues inventent la préhistoire. Publications scientifiques du Muséum, coll. « Archives », Paris, pp. 151-211.

13. Vibraye P. de (1859-1860) Note sur les ossements fossiles accompagnés d'une mâchoire humaine, trouvés dans les grottes d'Arcy-sur-Yonne. Bulletin de la Société géologique de France $2^{\mathrm{e}}$ série 17: 462-78.

14. Hurel A., Coye N. (2016) Moulin Quignon 1863-1864 : détours inédits et bilan historiographique. L'Anthropologie 120(4): 314-43.

15. Hurel A. (2007) La France préhistorienne, de la Révolution à 1941. CNRS Éditions, Paris, 284 p.

16. Lartet L. (1868) Sur une sépulture des anciens Troglodytes du Périgord. Revue des sociétés savantes $2^{\mathrm{e}}$ série 3: 279-87.

17. Lartet É., Christy H. (1864) Sur des figures d'animaux gravées ou sculptées et autres produits d'art et d'industrie rapportables aux temps primordiaux de la période humaine. Revue archéologique 9: 233-67.

18. Dupont É.-F. (1867) Étude sur les fouilles scientifiques exécutées pendant l'hiver de 1865-1866 dans les cavernes des bords de la Lesse [Bulletins de l'Académie royale de Belgique, $2^{\mathrm{e}}$ série, $\mathrm{t}$. XXII, $\left.n^{\circ} 7,1866\right]$. Notices préliminaires sur les fouilles exécutées sous les auspices du gouvernement belge dans les cavernes de la Belgique par M. Édouard Dupont, t. I, Muquardt et Cie, Bruxelles, pp. 3-26

19. Broca P. (1866) Discussion. Sur la mâchoire humaine de la Naulette. Bulletins de la Société d'anthropologie de Paris (1): 593-603

20. Dupont É.-F. (1867) Étude sur l'ethnographie de l'homme de l'âge du renne dans les cavernes de la vallée de la Lesse : ses caractères; sa race; ses industries; ses mœurs [Mémoires couronnés et autres mémoires publiés par l'Académie royale de Belgique, t. XIX, 1867]. Notices préliminaires sur les fouilles exécutées sous les auspices du gouvernement belge dans les cavernes de la Belgique par M. Édouard Dupont, t. II, Muquardt et Cie, Bruxelles, pp. 3-76.

21. Lartet É. (1864) Lettre relative aux fouilles exécutées à Bruniquel, adressée par M. Lartet à Son Excellence M. le Ministre de 1'Instruction publique. Revue des sociétés savantes 6: 321-6.

22. Ferry H., Arcelin A. (1868) L'âge du renne en Mâconnais, Mémoire sur la station du Clos du Charnier de Solutré. Imprimerie Émile Protat, Mâcon, 42 p.

23. Ferry H., Arcelin A. (1869) L'âge du renne en Mâconnais, Mémoire sur le gisement archéologique du Clos du Charnier à Solutré, département de Saône-et-Loire. International Congress of Prehistoric Archaeology: transactions of the third session which opened at Norwich on the $20^{\text {th }}$ August and closed in London on the $28^{\text {th }}$ august 1868 . Longmans, Green and Co, London : $319-50$.

24. Cartailhac É. (1872) Un squelette humain de l'âge du renne à Laugerie-Basse (Dordogne). Bulletin de la Société d'histoire naturelle de Toulouse 6: 204-26.

25. Lyell C. (1863) The Geological evidence of the antiquity of man with remarks on theories of the origin of species by variation, John Murray, London, 520 p.
26. Lyell C. (1864) L'ancienneté de l'homme prouvée par la géologie. J.B. Baillière et fils, Paris, $557 \mathrm{p}$.

27. Quatrefages A. de (1872) Les origines européennes. La race prussienne. La Revue scientifique de la France et de l'étranger : revue des cours scientifiques $2^{\mathrm{e}}$ série (2): 25-33

28. Mortillet G. de (1868) Station et sépultures de Solutré. Matériaux pour l'histoire primitive et philosophique de l'homme 4: 317-24.

29. Cartailhac É. (1872). Un squelette humain de l'âge du renne à Laugerie-Basse (Dordogne). Bulletin de la Société d'histoire naturelle de Toulouse 6: 204-26.

30. Fischer H. (1876). « Sur la conchyliologie des cavernes », Bulletins de la Société d'anthropologie de Paris, $2^{\mathrm{e}}$ série 11: 181-5.

31. Mortillet G. de (1884) Cro-Magnon (paléoethnologie). Dictionnaire des sciences anthropologiques. Octave Doin, Paris : 357

32. Foucault M. (1966) Les mots et les choses. Une archéologie des sciences humaines. Gallimard, Paris, 400 p.

33. Blanckaert C. (2009) Le Rapport sur les progrès de l'anthropologie d'Armand de Quatrefages. In : Barbin É., Godet J.-L., Stenger G. (ed) 1867. L'année de tous les rapports. Les lettres et les sciences à la fin du Second Empire. Éditions du Temps, Pornic, pp. 270-82.

34. Hamy E.T. (1868) La septième réunion des sociétés savantes à la Sorbonne. Deuxième article. La gazette médicale de Paris 20: 279-85

35. Broca P. (1866) Anthropologie. Dictionnaire encyclopédique des sciences médicales, t. 5 ANG-ARB [1870]: 276-300

36. Blanckaert C. (2009) De la race à l'évolution. Paul Broca et l'anthropologie française (1850-1900), Paris, L'Harmattan, coll. Histoire des sciences humaines, pp. 319-57

37. Richard N. (1989) La revue L'Homme de Gabriel de Mortillet. Anthropologie et politique au début de la Troisième république. Bulletins et Mémoires de la Société d'anthropologie de Paris 1 (3): 231-55.

38. Desmet P. (1996) La linguistique naturaliste en France (18671922). Nature, origine et évolution du langage, Peeters, LeuvenParis, $633 \mathrm{p}$.

39. Conry Y. (1974) L'introduction du darwinisme en France au $\mathrm{XIX}^{\mathrm{e}}$ siècle, Vrin, Paris, $480 \mathrm{p}$.

40. Retzius A. A. (1846) Mémoire sur les formes du crâne des habitants du Nord. Annales des sciences naturelles - Zoologie 6: 133-71.

41. Blanckaert C. (1989) L'indice céphalique et l'ethnogénie européenne : A. Retzius, P. Broca, F. Pruner-Bey (1840-1870). Bulletins et Mémoires de la Société d'anthropologie de Paris nouvelle série 1(3-4): 165-202

42. Nilsson S. (1848) On the Primitive Inhabitants of Scandinavia. Report of the seventeenth meeting of the British Association for the Advancement of Science, Oxford, 1847, John Murray, London : 31-21

43. Pruner-Bey F. (1865) Anciens crânes des types ligure et celtique. Bulletins de la Société d'anthropologie de Paris. 6: 458-74.

44. Pruner-Bey F. (1866) Étude et description de plusieurs crânes ligures. Bulletins de la Société d'anthropologie de Paris. $2^{\mathrm{e}}$ série 1: 442-67.

45. Broca P. (1868) Discours de M. Broca sur l'ensemble de la question. Congrès international d'anthropologie et d'archéologie préhistoriques. Compte rendu de la $2^{\mathrm{e}}$ session, Paris, 1867. Reinwald, Paris, pp. 367-402.

46. Broca P. (1868) Les crânes des Eyzies et la théorie esthonienne. Bulletins de la Société d'anthropologie de Paris. $2^{\mathrm{e}}$ Série 3 : 454-514.

47. Broca P. (1868) Sur les crânes et ossements des Eyzies. Bulletins de la Société d'anthropologie de Paris. $2^{\mathrm{e}}$ Série 3: 350-92.

48. Tylor E. B. (1869) The condition of prehistoric races, as inferred from observation of modern tribes. International Congress of Prehistoric Archaeology: transactions of the third session which opened at Norwich on the $20^{\text {th }}$ August and closed in London on the $28^{\text {th }}$ august 1868 . Longmans, Green and Co, London : 11-26. 